\title{
On the possibility of enrichment and differentiation in gas giants during birth by disk instability
}

\author{
Aaron C. Boley ${ }^{1}$ and Richard H. Durisen ${ }^{2}$ \\ ${ }^{1}$ Department of Astronomy; University of Florida, 211 Bryant Space Science Center, \\ Gainesville, FL 32611, USA \\ email:aaron.boley@gmail.com \\ ${ }^{2}$ Department of Astronomy, Indiana University, 727 East 3rd Street, Swain West 319, \\ Bloomington, IN 47405, USA
}

\begin{abstract}
We investigate the coupling between solids and gas during the formation of gas giant planets by disk fragmentation in the outer regions of massive disks. We find that fragments can become differentiated at birth. Even if an entire clump does not survive, differentiation could create solids cores that survive to accrete gaseous envelopes later.
\end{abstract}

Keywords. planetary systems: protoplanetary disks, planetary systems: formation

A gravitationally unstable disk can be very efficient at concentrating solids in spiral arms (Rice et al. 2004, 2006). If fragmentation does occur, clumps form from small sections of these arms (Durisen et al. 2008; Boley et al. 2010), exactly where solids are aerodynamically captured. These circumstances can lead to enrichment and differentiation in fragments at birth. To explore this mechanism, we have run a number of hydrodynamics simulations, exploring different initial conditions, opacities, and particle sizes. In this summary, we highlight the differentiation results from Boley \& Durisen (2010), focusing on the simulation called SIM2, which explores $10 \mathrm{~cm}$ and $1 \mathrm{~km}$-size particles separately. The disk is the same as SIMD in Boley (2009), which surrounds a $1 \mathrm{M}_{\odot}$ star and has a disk mass that reaches $0.33 \mathrm{M}_{\odot}$ through envelope accretion. The re-simulation is begun about $1 / 2$ orbit, measured at the fragmentation radius, before clump formation. Particles are distributed to give a solid-to-gas ratio of approximately 0.01 everywhere.

Rock-size $(10 \mathrm{~cm})$ particles show a high degree of concentration in the spiral arms prior to fragmentation (Fig. 1). However, the clump also forms from surrounding solid-depleted gas, diluting the overall enrichment to about 1.4 over the nebular value. As the clump evolves and grows, this ratio is roughly maintained as the clump feeds through solidenhanced spiral wakes. Although there is only modest enrichment overall, the clump experiences a high degree of differentiation immediately after birth (see Table 1). In contrast, the simulation with only km-size solids shows an overall depletion of heavy elements in the clump because the solids cannot dissipate kinetic energy and contract with the gas. The degree of enrichment and differentiation following fragmentation will depend on the size distribution of solids, which can lead to a variety of outcomes and potential core sizes.

Some clumps may survive to form planets or brown dwarfs directly (Cameron 1978; Boss 1998), while others will be destroyed through, e.g., tidal disruption (Boley et al. 2010). In both cases, large cores can form. If the solid core is separated from its nascent clump, it may still undergo a subsequent phase of rapid gas accretion. Because the core 
is formed rapidly in a clump, we call this scenario core assist plus gas capture. For further results and discussions, see Boley \& Durisen (2010).

Table 1. Gas and rock mass for the clump that forms in SIM2 with $10 \mathrm{~cm}$ and km-size solids. Three different density thresholds are shown, where only mass above the threshold is considered. Full represents the entire mass of the clump, here for all gas that has $\rho>9 \times 10^{-13} \mathrm{~g} \mathrm{~cm}^{-3}$ and for $T>34 \mathrm{~K}$. Half refers to all mass that is above half of the peak density in the clump, $\rho_{\max }$, and $\gtrsim 90 \%$ is for only mass that is greater than $90 \%$ of the peak. This last threshold isolates the core conditions of the clump. The factor $f_{\mathrm{RtoG}}$ gives the ratio of rock mass to gas mass, and $f_{\text {enr }} \equiv\left(f_{\text {RtoG }}+0.01\right) / 0.02$ gives the total solids enrichment relative to the average nebula's value. When we write "rocks," we are referring to a mixture of silicates and ices.

\begin{tabular}{lllll}
\hline SIM2 $10 \mathrm{~cm}$ & Time $=1450 \mathrm{yr}$ & $r \sim 104 \mathrm{AU}$ & $\rho_{\max }=1.7 \times 10^{-11} \mathrm{~g} \mathrm{~cm}^{-2}$ & \\
\hline Density Threshold & Gas $\left(M_{J}\right)$ & Rocks $\left(M_{\oplus}\right)$ & $f_{\text {RtoG }}$ & $f_{\text {enr }}$ \\
\hline Full & 8.1 & 42 & 0.016 & 1.3 \\
Half & 3.5 & 38 & 0.034 & 2.2 \\
$\gtrsim 90 \%$ & 0.85 & 32 & 0.12 & 6.4 \\
\hline SIM2 km & Time $=1430$ yr & $r \sim 106 \mathrm{AU}$ & $\rho_{\max }=1.3 \times 10^{-11} \mathrm{~g} \mathrm{~cm}^{-2}$ & \\
\hline Full & 7.1 & 7.2 & 0.0032 & 0.66 \\
Half & 3.7 & 2.6 & 0.0022 & 0.61 \\
$\gtrsim 90 \%$ & 1.3 & 0.67 & 0.0016 & 0.58 \\
\hline
\end{tabular}
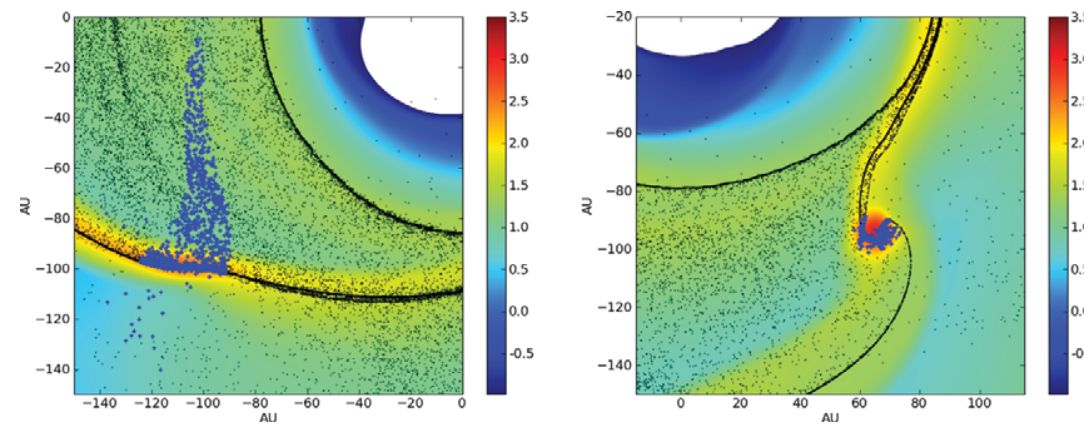

Figure 1. The spiral arm in SIM2 just before fragmentation at $960 \mathrm{yr}$ (left) and after fragmentation at the $1300 \mathrm{yr}$ snapshot (right). Gas density is shown by the colorbar, while solids are given by black dots. The rocks that are considered to be part of the clump at $1300 \mathrm{yr}$ are shown with large, blue crosses. The same particles are shown in the $960 \mathrm{yr}$ snapshot. Most of the mass comes from a 20-30 AU section of the spiral arm, consistent with Durisen et al. (2008) and Boley et al. (2010). The solids are highly concentrated near the peak gas density, which is slightly offset from the geometric center in projection.

\section{References}

Boley, A. C. 2009, ApJ, 695, 53L

Boley, A. C., \& Durisen, R. H. 2010, ApJ, 724, 618

Boley, A. C., Hayfield, T., Mayer, L., \& Durisen, R. H. 2010, Icarus, 207, 509

Boss, A. P. 1998, ApJ, 503, 923

Cameron, A. G. W. 1978, Moons and the Planets, 18, 5

Durisen, R. H., Hartquist, T. W., \& Pickett, M. K. 2008, Apध्धSS, 317, 3D

Rice, W. K. M., Lodato, G., Pringle, J. E., Armitage, P. J., \& Bonnell, I. A. 2004, MNRAS, 355,543

-. 2006, MNRAS, 372, L9 